\title{
Determination of calculation parameters for difference approximation of group refractive index of air based on two-point central method
}

\author{
Dong Wei ${ }^{1^{*}}\left(\mathbb{D}\right.$, Muzheng Xiao ${ }^{2}$ and Ping Yang ${ }^{3}$
}

\begin{abstract}
Background: The accurate knowledge of the refractive index of air is very important for environmental compensation in length measurements.

Methods: In this study, we apply difference approximation methods to facilitate the calculation of the group refractive index of air (GRA) over all calculable wavelengths. Our approach involves the determination of a suitable combination of the step size $\Delta \lambda$ and numbers of significant digits of calculations of the phase refractive index of air (PRA) by balancing the two main errors (round-off and truncation errors) over the entire calculable wavelength range.

Results: Based on our calculations, we find that the GRA computation over the range of all calculable wavelengths (301.5-1698.5 nm) can be easily approximated by the two-point central difference method with $\Delta \lambda=1.5 \mathrm{~nm}$ and 12digit PRA accuracy.

Conclusion: The approximation accuracy is less than $5 \times 10^{-9}$. Our approach can be used by non-expert users to obtain the GRA with sufficient accuracy.
\end{abstract}

Keywords: Group refractive index, Difference approximation, Frequency comb, Length measurement, Metrology

\section{Background}

The accurate knowledge of the refractive index of air is very important in length measurements. The wavelength of a laser (which is commonly used for length measurements) propagating in air changes depending on the air refractive index. A lower refractive index of air corresponds to a higher speed of light, and as the speed of light changes, its wavelength also changes. When measuring linear displacements in air with a laser, it is particularly necessary to perform wavelength compensation. For most applications, the refractive index of air is directly calculated by the Edlén equation (for e.g., [1-4]).

Here, we consider the case of a length measurement based on the use of the wavelength of a single-mode laser. Firstly, we determine values for air pressure,

\footnotetext{
* Correspondence: weidong@mech.nagaokaut.ac.jp

'Department of Mechanical Engineering, Nagaoka University of Technology, Nagaoka City, Niigata 940-2188, Japan

Full list of author information is available at the end of the article
}

temperature, and humidity using sensors. We next calculate the phase refractive index of air (PRA) as $n_{p}$. Secondly, we know the laser vacuum wavelength to be $\lambda_{\text {vac }}$, and therefore, using $n_{p}$ and $\lambda_{v a c}$, we calculate the laser wavelength in air, $\lambda_{\text {air }}$. For a linear displacement $\mathrm{L}$, we measure $L$ as $L_{\text {air }}=\lambda_{\text {air }} \times(M+N) / 2$. Here, $M$ and $N$ denote the integer and fraction parts, respectively, of the length measurement. Next, it is required to compensate for the influence of $n_{p}$ on $L_{\text {air }}$. By calculating $L_{v a c}=L_{a i r} \times n_{p}$, we can convert the length measured in air $\left(\mathrm{L}_{\text {air }}\right)$ to the length in vacuum, $L_{\mathrm{vac}}$. Without the influence of $n_{p}$, the values of lengths in vacuum are comparable with each other.

Probably the most exciting recent advancement in the field of length measurement is the development of the distance ranging technique based on the femtosecond optical frequency comb (FOFC) [5]. This technique enables us to measure length based on not only wavelength (e.g., [6-9]) but also the adjacent pulse repetition 
interval length (APRIL) (e.g., [10-20]), where APRIL denotes the physical length between two adjacent pulses.

In the study, we consider the case of a length measurement based on the use of the APRIL of an FOFC laser. We acquire values for air pressure, temperature, and humidity, and we calculate the group refractive index of air (GRA), $\mathrm{n}_{\mathrm{g}}$, using the following equation [21].

$$
\begin{aligned}
\mathrm{n}_{\mathrm{g}}\left(\lambda_{\text {cen_vac }}\right) & =\mathrm{n}_{\mathrm{p}}\left(\lambda_{\text {cen_vac }}\right)-\lambda_{\text {cen_vac }} \\
& \times\left(\mathrm{d} \mathrm{n}_{\mathrm{p}}\left(\lambda_{\text {vac }}\right) / \mathrm{d} \lambda_{\text {vac }}\right)_{\lambda_{\text {cen_vac }}},
\end{aligned}
$$

where $\lambda_{\text {cen_vac }}$ represents the central wavelength of the FOFC. Further, $\left(\operatorname{dn}_{\mathrm{p}}\left(\lambda_{\mathrm{vac}}\right) / \mathrm{d} \lambda_{\mathrm{vac}}\right)_{\lambda_{\text {cenvac }}}$ represents the derivative of the function $\mathrm{y}=\mathrm{n}_{\mathrm{p}}\left(\lambda_{\mathrm{vac}}\right)$ at $\lambda_{\mathrm{vac}}=\lambda_{\text {cen_vac. }}$.

We know that the vacuum value of APRIL is $\Lambda_{\mathrm{vac}}$. Using $\mathrm{n}_{\mathrm{p}}$ and $\Lambda_{\mathrm{vac}}$, we calculate the FOFC's APRIL in air, $\Lambda_{\text {air }}$. For a linear displacement $\mathrm{L}$, we represent $\mathrm{L}$ as $\mathrm{L}_{\Lambda \text { air }}$ $=\Lambda_{\text {air }} \times\left(\mathrm{M}_{\Lambda}+\mathrm{N}_{\Lambda}\right) / 2$. Here, $\mathrm{M}_{\Lambda}$ and $\mathrm{N}_{\Lambda}$ denote the integer and fraction parts, respectively, of the length measurement, and they are required when $\mathrm{L}_{\Lambda \text { air }}$ is measured in units of $\Lambda_{\text {air }}$. Next, we compensate for the influence of $\mathrm{n}_{\mathrm{g}}$ on $\mathrm{L}_{\Lambda \text { air }}$. By calculating $\mathrm{L}_{\Lambda \mathrm{vac}}=\mathrm{L}_{\Lambda \text { air }} \times \mathrm{n}_{\mathrm{g}}$, we can convert the length measured in air $\mathrm{L}_{\Lambda \text { air }}$ to the length in vacuum $L_{\Lambda \text { vac }}$. Without the influence of $n_{g}$, the values of the lengths in vacuum are comparable with each other.

In essence, for APRIL-based distance evaluation, the GRA is required, which can be calculated via the derivative of the PRA with respect to the wavelength.

The expression of the PRA given by the empirical formula is a complicated function, and mistakes in differentiation can lead to transformation and calculation mistakes. One way to avoid this problem is to use the difference approximation. The differential approximation is a method of approximating derivatives with algebraic operation of discrete values. In a previous research [22], we determined that by means of the two-point central difference with a step size of $10 \mathrm{~nm}$, the GRA could approximated by an order of $60 \times 10^{-9}$ for both the visible light range (380-770 $\mathrm{nm}$ ) and the optical fiber light transmittance range (1260-1625 $\mathrm{nm})$. In addition [23], we found that the GRA computation can be easily approximated by the four-point central difference approximation method and that approximation accuracies of less than $30 \times 10^{-9}$ can be achieved over the entire wavelength range (320-1680 nm) with a step size of $10 \mathrm{~nm}$.

The question remains as to whether the applications of the two-point difference approximation could be sufficiently accurate to cater to non-expert users over the entire abovementioned wavelength range. When compared with the four-point central difference approximation method, the calculation of two-point difference approximation method is very simple. In previous researches [22, 23], we calculated the PRA with nine-digit accuracy. In this study, we verify the achievable accuracy of the two-point difference approximation method for different step sizes and number of significant digits (NSD) of the PRA.

The rest of the paper is organized as follows. Section 2 presents an overview of the definition of two-point difference approximation. Section 3 presents our numerical calculations. Section 4 presents the results of our numerical calculations and discussions. Finally, Section 5 presents our conclusions.

\section{Methods}

Having understood the importance of environmental compensation in length measurement, we next focus our attention on differential calculations by difference approximations (e.g., [24, 25]). The complexity of the calculation of the two-point difference methods (namely, two-point forward, two-point backward, and two-point central difference methods) is the same. As can been inferred from previous researches [22, 23], when compared with the two-point central difference method, no significant improvement in the calculation accuracy can be expected with the two-point forward and two-point backward difference methods. Based on these two facts, we only consider the two-point central difference method in the following discussion. We first present the difference approximation equations for the convenience of users. The expression for the GRA calculated by means of the two-point central $\mathrm{n}_{\mathrm{p} \_2 \text { cent }}\left(\lambda_{\mathrm{vac}}\right)$ difference method is presented as Eqs. (2).

$$
\begin{aligned}
\mathrm{n}_{g_{\text {_cent }}}\left(\lambda_{\mathrm{vac}}\right)= & \mathrm{n}_{\mathrm{p}}\left(\lambda_{\mathrm{vac}}\right)-\lambda_{\text {vac }} \\
& \times\left[\frac{\mathrm{n}_{\mathrm{p}}\left(\lambda_{\mathrm{vac}}+\Delta \lambda\right)-\mathrm{n}_{\mathrm{p}}\left(\lambda_{\mathrm{vac}}-\Delta \lambda\right)}{2 \times \Delta \lambda}\right]
\end{aligned}
$$

With this understanding of the calculation of $n_{g}$ for environmental compensation and the concept of the difference approximation, we turn to the numerical calculations.

The goal of this study is to determine a specific step size and NSD of $n_{p}$ that can be applied to most wavelengths, thereby resulting in an approximated calculation of $n_{g}$ with a negligible difference from the actual value. The Edlén formula affords an accuracy of approximately $3 \sim 5 \times 10^{-8}$ [26]. We set a difference of $<5 \times 10^{-9}$ between differential calculations and the differential approximation as an acceptable level of difference.

Due to length constraints of this paper, we only discuss the Edlén empirical equations [2-4] in which the PRA can be derived as a function of the wavelength $\lambda_{\mathrm{vac}}$, temperature $T$, barometric pressure $P$, and humidity $H$. For the phase refractive index, we use the equations given in Ref. [4]. 


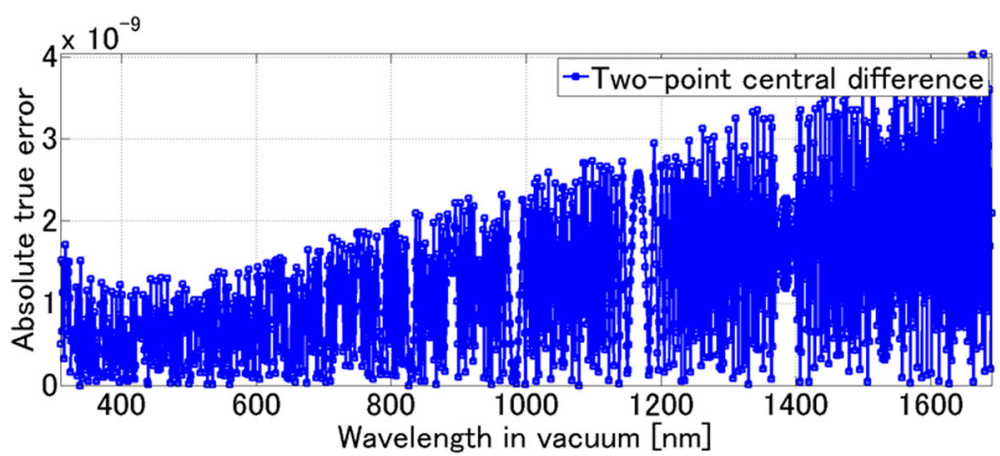

Fig. 1 Variation in the absolute value of difference as a function of wavelength for $\Delta \lambda=1 \mathrm{~nm}$ upon calculating the phase refractive index of air (PRA) with 12-digit accuracy

\section{Numerical experiments}

The absolute value of (the) difference (between the theoretical and approximated values) is a function of the approximation method, step size, differential wavelength point, and NSD of $\mathrm{n}_{\mathrm{p}}$. The calculable range of the empirical formula [4] is 300 to $1700 \mathrm{~nm}$, which means that $\lambda_{\text {vac }}+\Delta \lambda$ and/or $\lambda_{\text {vac }}-\Delta \lambda$ must lie within this range. In other words, for the two-point central difference method, the calculable ranges of the GRA is $\left[\lambda_{\min }, \lambda_{\max }\right]$ ( $\left.\lambda \max +\Delta \lambda \leq 1700, \lambda_{\min }-\Delta \lambda \geq 300\right)$. Since our objective is the determination of a specific step size that can be applied to almost all wavelengths, as a test, we apply step sizes that are multiples of 10 and less than $10 \mathrm{~nm}$.

In the study, the first numerical calculations were performed under the following environmental conditions: $T=20^{\circ} \mathrm{C}, P=101.325 \mathrm{kPa}$, and $H=50 \%$. The theoretical value of $\mathrm{n}_{g}\left(\lambda_{\mathrm{vac}}\right)\left(\lambda_{\mathrm{vac}}: 300 \leq \lambda_{\mathrm{vac}} \leq 1700 \mathrm{~nm}\right)$ was calculated with the use of Eq. (1). The approximate values of $\mathrm{n}_{g_{-} 2 \text { cent }}\left(\lambda_{\text {vac }}\right)$ were calculated based on Eqs. (2), for different $\lambda_{\text {vac }}$ values. Subsequently, by using these values, we calculated the absolute value of the difference between the theoretical and difference approximation values as $\left|\mathrm{n}_{g}\left(\lambda_{\text {vac }}\right)-\mathrm{n}_{g_{-} 2 \text { cent }}\left(\lambda_{\text {vac }}\right)\right|$ for the two-point central difference method. The approximate values change when the step sizes and NSD of the PRA change.

\section{Results and discussion}

Figure 1 shows the variation in the absolute value of difference with the calculable wavelength with $\Delta \lambda=1 \mathrm{~nm}$ upon calculating the PRA with 12-digit accuracy. In Fig. 1, we note that the maximum absolute value of the twopoint central difference method is obtained at around $1700 \mathrm{~nm}$. As mentioned in a previous study [23], the "zigzag" nature of the curves is due to the round-off error in calculating the PRA. The round-off error originates from numerical calculations that use a limited number of digits in a computer. In the case of this combination (namely, $\Delta \lambda=1 \mathrm{~nm}$ and $\mathrm{n}_{\mathrm{p}}$ with 12 digits), the absolute value of the difference of the two-point central difference method is mainly affected by the round-off error. The reason underlying the high round-off error occurring at larger wavelengths is that the curve of PRA at larger wavelengths is "gentler" than that at smaller wavelengths. A gentle curve means the difference between these two values used for calculation is too small. If we do not consider more digits, we cannot accurately evaluate their difference.

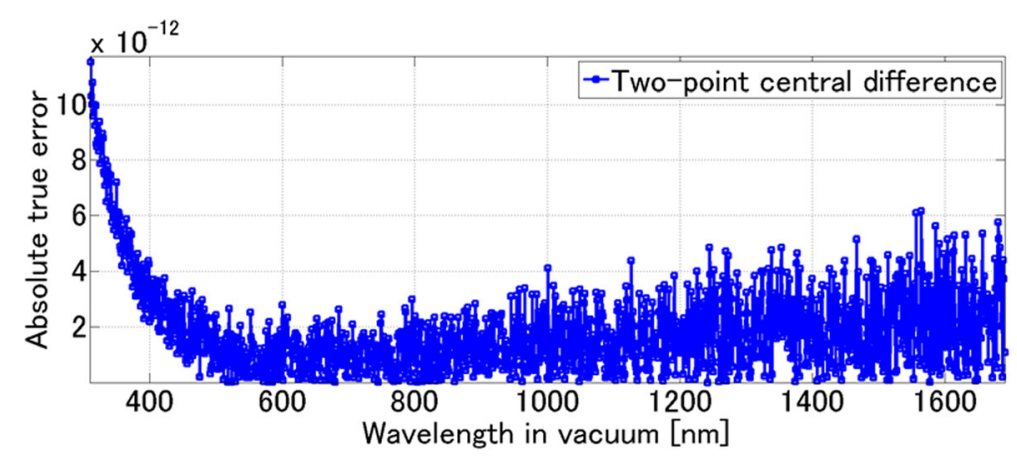

Fig. 2 Variation in the absolute value of difference as a function of wavelength for $\Delta \lambda=0.1 \mathrm{~nm}$ upon calculating the phase refractive index of air (PRA) with 16-digit accuracy 


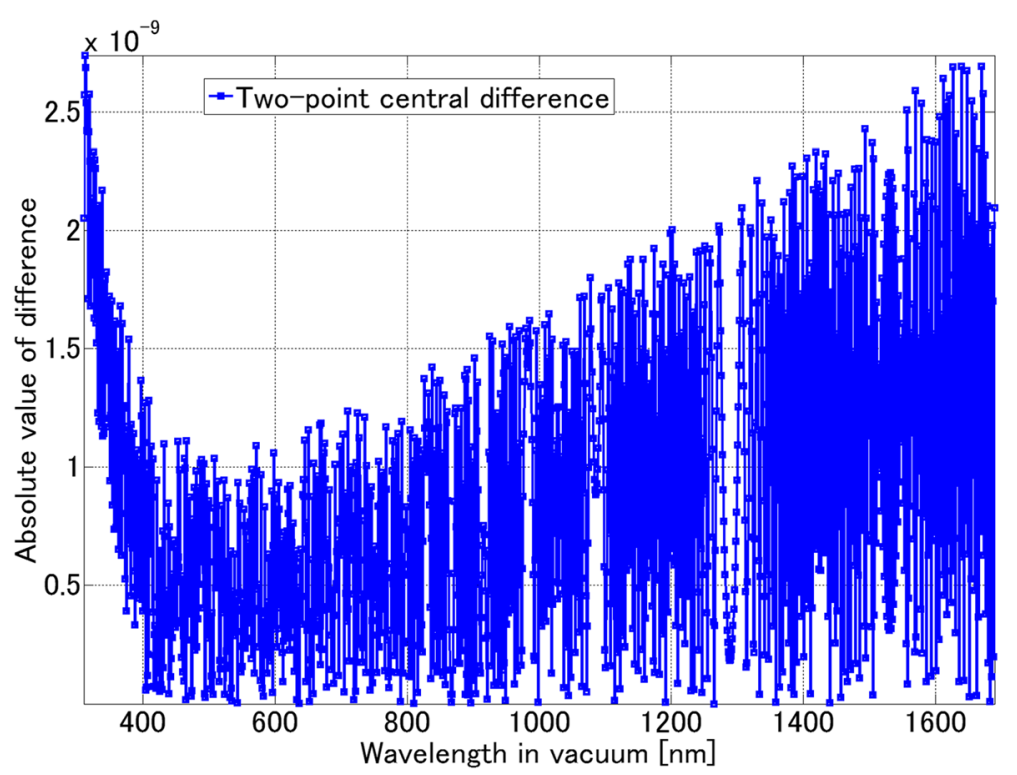

Fig. 3 Variation in the absolute value of difference obtained with the two-point central difference method as a function of wavelength for $\Delta \lambda=1.5 \mathrm{~nm}$ upon calculating the phase refractive index of air (PRA) with 12-digit accuracy

We next change the step size $\Delta \lambda$ and NSD of the PRA to observe the change in the maximum absolute value of difference. For example, Fig. 2 shows the variation in the absolute value of difference with $\Delta \lambda=0.1 \mathrm{~nm}$ upon calculating the PRA with 16-digit accuracy. From the figure, we note that the maximum absolute value of difference obtained with the two-point central difference method exhibits a corresponding change, with the corresponding position shifting to around $300 \mathrm{~nm}$. The absolute values of difference obtained at smaller wavelengths are mainly affected by the truncation error. Truncation error exists because the difference approximation is calculated based on the linear Taylor expansion. This shift of the maximum value indicates that the main error contributor is now the truncation error and not the roundoff error. In the case of this combination (namely, $\Delta \lambda=$ $0.1 \mathrm{~nm}$ and $\mathrm{n}_{\mathrm{p}}$ with 16 digits), the maximum absolute value of difference for the two-point central difference method is mainly affected by the truncation error. The reason underlying high truncation error at smaller wavelengths is that the curve of the PRA at smaller wavelengths is "steeper" than that at larger wavelengths.

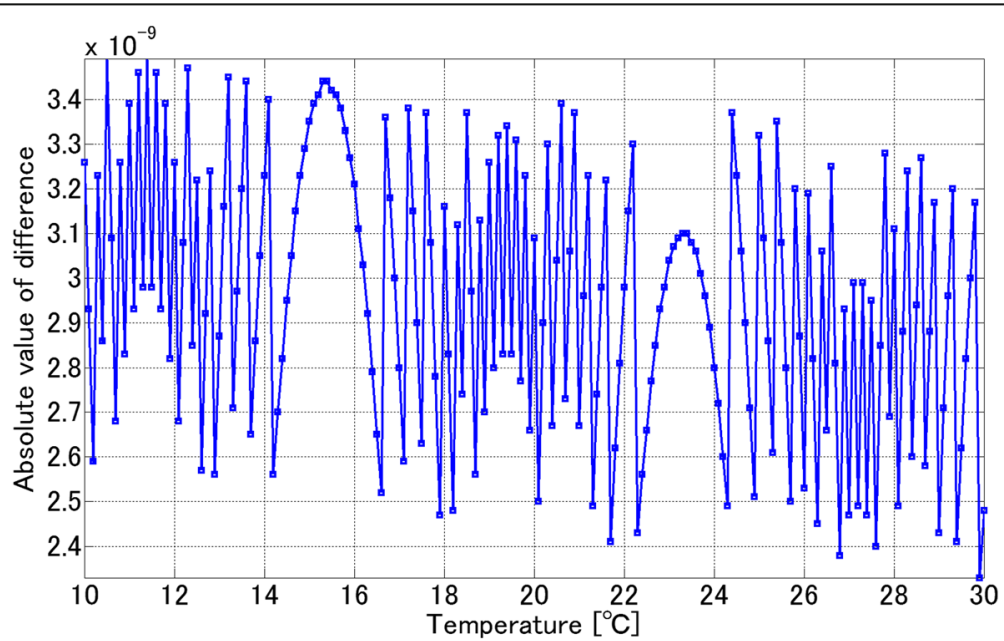

Fig. 4 Variation in the absolute value of difference obtained the with two-point central difference method as a function of temperature $\left(\lambda_{\text {vac }}=301.5 \mathrm{~nm}\right.$, $P=101.325 \mathrm{kPa}, H=50 \%)$ 


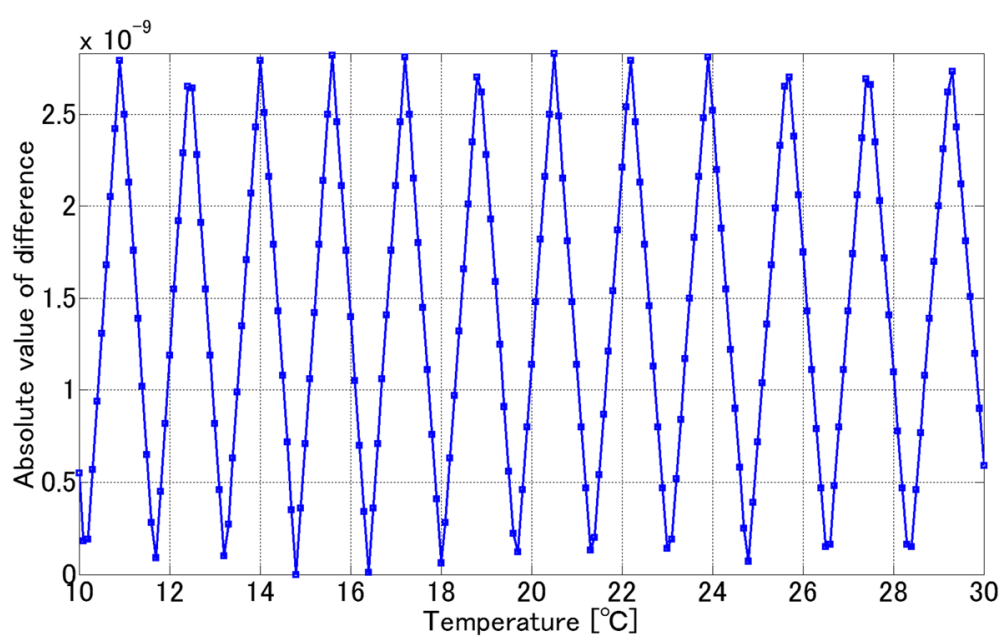

Fig. 5 Variation in the absolute value of difference obtained with two-point central difference method as a function of temperature $\left(\lambda_{\mathrm{vac}}=1698.5 \mathrm{~nm}\right.$, $P=101.325 \mathrm{kPa}, \mathrm{H}=50 \%)$

Since the difference approximation is a linear approximation, its precision is suitable for a gentle curve, and its accuracy is poor for a steep curve.

When the combination of $\Delta \lambda$ and the NSD of PRA is changed, the main contributor of the error in the maximum absolute value of difference also changes. It is difficult to estimate the absolute value of difference based on the law of propagation of uncertainty. For this reason, we perform numerical simulations to evaluate the maximum absolute value of difference without considering the main cause of error. Here, we remark that the PRA is a continuous function, and therefore, we only test the range around the upper and lower limits of the variation range of the environmental parameters. Since the maximum absolute value of the difference is obtained around $300 \mathrm{~nm}$ and $1700 \mathrm{~nm}$, we address only these two cases in the following discussion.

Figures 1 and 2 indicate that there is an optimal combination of $\Delta \mathrm{l}$ and NSD of PRA that can minimize the total error. We can determine a suitable estimate of this combination by balancing the truncation error and the round-off error of the PRA; this forms the basis of our strategy. We test different pairs of $\Delta \lambda$ and PRA digits. Figure 3 shows a candidate result that can be optimized to almost all wavelengths with $\Delta \mathcal{l}=1.5 \mathrm{~nm}$ and 12-digit PRA accuracy. We note that the maximum absolute value of difference is 2 . $7 \times 10^{-9}$. Since the purpose of this research is to determine a combination of the step size $\Delta \lambda$ and NSD of the PRA satisfying the error conditions, we only consider this combination in the following discussion.

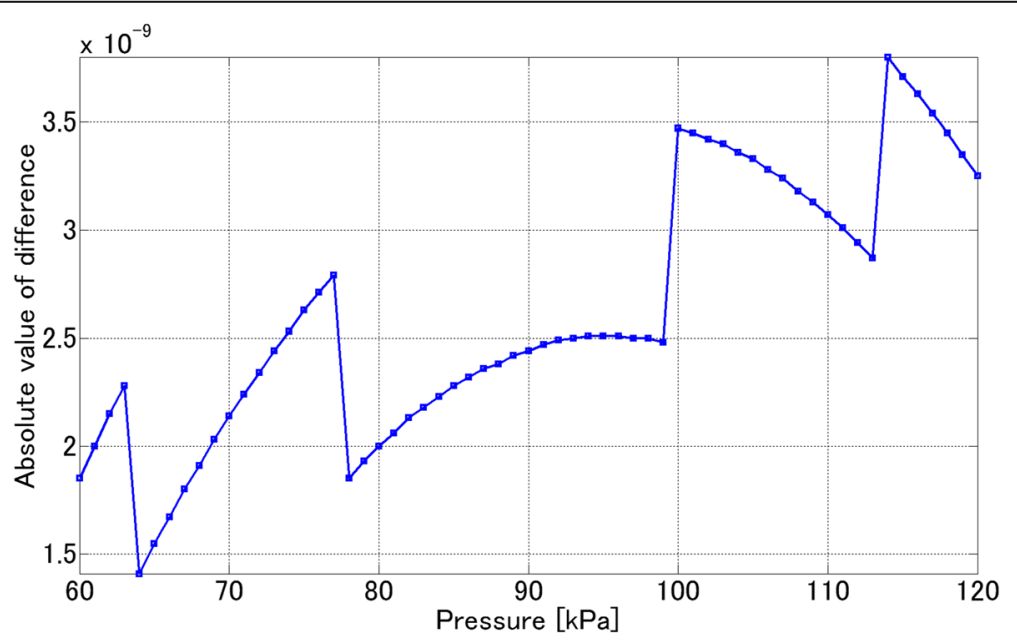

Fig. 6 Variation in the absolute value of difference obtained with the two-point central difference method as a function of pressure $\left(\lambda_{\text {vac }}=301.5 \mathrm{~nm}\right.$, $\left.T=10^{\circ} \mathrm{C}, H=50 \%\right)$ 


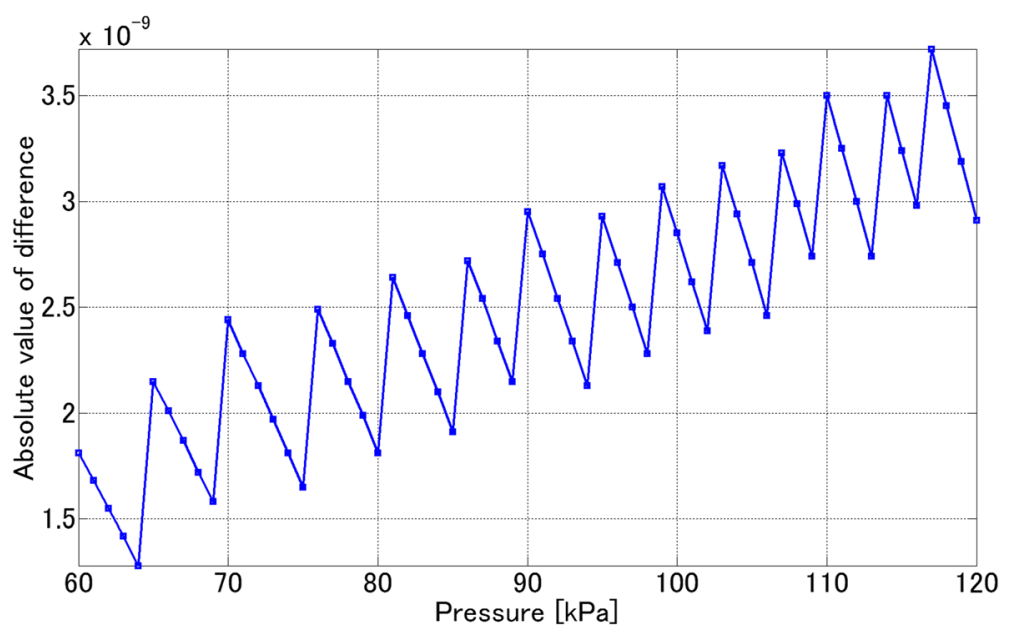

Fig. 7 Variation in the absolute value of difference obtained with the two-point central difference method as a function of pressure $\left(\lambda_{\text {vac }}=301.5 \mathrm{~nm}\right.$, $\left.T=30{ }^{\circ} \mathrm{C}, H=50 \%\right)$

Figures 4 and 5 show the variation in the absolute value of difference as a function of temperature. The maximum absolute values of difference in Figs. 4 and 5 are $3.5 \times 10^{-9}$ and $2.8 \times 10^{-9}$, respectively. The maximum absolute value of difference with $\lambda_{\mathrm{vac}}=301.5 \mathrm{~nm}$ is larger than that of $\lambda_{\text {vac }}=1698.5 \mathrm{~nm}$. In the following discussion, we only present the results for $\lambda_{\text {vac }}=301.5 \mathrm{~nm}$.

Figures 6 and 7 depict the variation in the absolute value of difference with pressure. The maximum absolute values of difference in Figs. 6 and 7 are $3.8 \times 10^{-9}$ and $3.7 \times 10^{-9}$, respectively. The maximum absolute value of difference at $T=10^{\circ} \mathrm{C}$ is larger than that at $T=$ $30{ }^{\circ} \mathrm{C}$, and therefore, in the following discussion, we subsequently address only the case of $T=10{ }^{\circ} \mathrm{C}$.
Figure 8 shows the variation in the absolute value of difference as a function of humidity. The maximum absolute value of difference in Fig. 8 is $4.1 \times 10^{-9}$.

Here, we again recall that the PRA is a continuous function. The maximum or minimum value of the function appears at the upper and lower bounds of the variable range. The round-off error leads to the zigzagging of the curve. As can be observed in the above figures, there is no significant deviation in the curve. Based on these facts, we can conclude that under environmental conditions of $T$ : $10 \leq T \leq 30{ }^{\circ} \mathrm{C}, P: 60 \leq P \leq 120 \mathrm{kPa}$, and $H: 5 \leq H \leq 85 \%$, the maximum absolute value of difference is less than $5 \times$ $10^{-9}$ over the entire wavelength range (301.5-1698.5 nm). This result proves the feasibility of our approach.

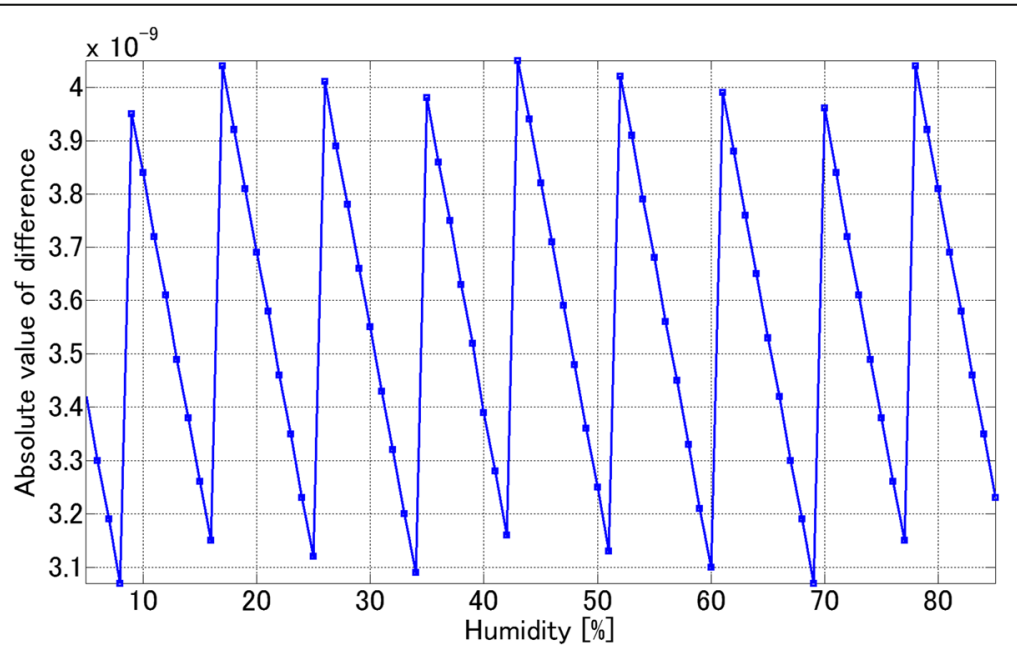

Fig. 8 Variation in the absolute value of difference obtained with the two-point central difference method as a function of humidity $\left(\lambda_{\mathrm{vac}}=301.5 \mathrm{~nm}\right.$, $\left.T=10^{\circ} \mathrm{C}, P=120 \mathrm{kPa}\right)$ 


\section{Conclusion}

Under environmental conditions of $T: 10 \leq T \leq 30{ }^{\circ} \mathrm{C}, P$ : $60 \leq P \leq 120 \mathrm{kPa}$, and $H: 5 \leq H \leq 85 \%$, we confirmed the accuracy of the two-point central difference approximation method via numerical calculations. Our strategy was to determine a suitable combination of the step size $\Delta \lambda$ and NSD of PRA by balancing the truncation error and the round-off error. We determined that the GRA computation can be easily approximated by the two-point central difference approximation method and that approximation accuracies of less than $5 \times 10^{-9}$ can be achieved over the entire wavelength range (301.5-1698.5 $\mathrm{nm}$ ) with $\Delta \mathrm{d}=1.5 \mathrm{~nm}$ and 12-digit PRA accuracy. Our method can be used by nonexpert users to obtain the GRA with sufficient accuracy for environmental compensation in length measurements.

\section{Abbreviations}

APRIL: Adjacent pulse repetition interval length; FOFC: Femtosecond optical frequency comb; GRA: Group refractive index of air; NSD: Number of significant digits; PRA: Phase refractive index of air

\section{Acknowledgments}

This research work was partially financially supported by Japan Society for the Promotion of Science (JSPS) KAKENHI Grant-in-Aid for Young Scientists (B) (Grant Number 17 K17743)

\section{Funding}

Declared at acknowledgements.

\section{Availability of data and materials}

Presented in the main paper.

\section{Authors' contributions}

WD proposed the idea, carried out the experiments and wrote the manuscript. All authors participated in the discussion of the experiments. All authors read and approved the final manuscript.

\section{Authors' information}

Dr. Dong WEI received his BSC and MSc in Engineering from the University of Electro-Communications of Japan, in 2006 and 2008, respectively, and he received his DSc from the university of Tokyo, Japan, in 2011. He is now an assistant professor in the Department of Mechanical Engineering at Nagaoka University of Technology in Japan. His interest includes applied optics and intelligent signal processing

\section{Competing interests}

The authors declare that they have no competing interests.

\section{Publisher's Note}

Springer Nature remains neutral with regard to jurisdictional claims in published maps and institutional affiliations.

\section{Author details}

${ }^{1}$ Department of Mechanical Engineering, Nagaoka University of Technology, Nagaoka City, Niigata 940-2188, Japan. ${ }^{2}$ School of Mechanical Engineering, Beijing Institute of Technology, Beijing 100081, People's Republic of China. ${ }^{3}$ Department of Mechanical and Electrical Engineering, School of Aerospace Engineering, Xiamen University, Xiamen City, Fujian Province 361006 People's Republic of China.

Received: 14 January 2018 Accepted: 2 May 2018 Published online: 10 May 2018

\section{References}

1. Ciddor, P.E.: Refractive index of air: new equations for the visible and near infrared. Appl. Opt. 35, 1566-1573 (1996).
2. Birch, K.P., Downs, M.J.: An updated Edlén equation for the refractive index of air. Metrologia. 30, 155 (1993).

3. Bengt, E.: The refractive index of air. Metrologia. 2, 71 (1966).

4. Stone JA and Zimmerman JH, Refractive index of air calculator. http:// emtoolbox.nist.gov/Wavelength/Edlen.asp.

5. Ye, J., Cundiff, S.T.: Femtosecond Optical Frequency Comb : Principle, Operation, and Applications, p. 361. Springer, New York, NY (2005), xii)

6. Schuhler, N., Salvade, Y., Leveque, S., Dandliker, R., Holzwarth, R.: Frequencycomb-referenced two-wavelength source for absolute distance measurement. Opt. Lett. 31, 3101-3103 (2006)

7. Hyun, S., Kim, Y.-J., Kim, Y., Jin, J., Kim, S.-W.: Absolute length measurement with the frequency comb of a femtosecond laser. Meas. Sci. Technol. 20, 095302 (2009)

8. Yasui, T., Kabetani, Y., Ohgi, Y., Yokoyama, S., Araki, T.: Absolute distance measurement of optically rough objects using asynchronous-opticalsampling terahertz impulse ranging. Appl. Opt. 49, 5262-5270 (2010).

9. Yokoyama, S., Yokoyama, T., Hagihara, Y., Araki, T., Yasui, T.: A distance meter using a terahertz intermode beat in an optical frequency comb. Opt. Express. 17, 17324-17337 (2009).

10. Yamaoka, Y., Minoshima, K., Matsumoto, H.: Direct measurement of the group refractive index of air with interferometry between adjacent femtosecond pulses. Appl. Opt. 41, 4318-4324 (2002).

11. Ye, J.: Absolute measurement of a long, arbitrary distance to less than an optical fringe. Opt. Lett. 29, 1153-1155 (2004).

12. Cui, M., Schouten, R.N., Bhattacharya, N., Berg, S.A.: Experimental demonstration of distance measurement with a femtosecond frequency comb laser. J. Eur. Opt. Soc. Rapid Publ. 3, 08003 (2008).

13. Matsumoto, H., Wang, X., Takamasu, K., Aoto, T.: Absolute measurement of baselines up to $403 \mathrm{~m}$ using heterodyne temporal coherence interferometer with optical frequency comb. Appl. Phys. Express. 5, 046601 (2012).

14. Narin, C., Satoru, T., Kiyoshi, T., Hirokazu, M.: A new method for highaccuracy gauge block measurement using $2 \mathrm{GHz}$ repetition mode of a mode-locked fiber laser. Meas. Sci. Technol. 23, 054003 (2012).

15. Wang, X., Takahashi, S., Takamasu, K., Matsumoto, H.: Space position measurement using long-path heterodyne interferometer with optical frequency comb. Opt. Express. 20, 2725-2732 (2012)

16. Wang, X., Takahashi, S., Takamasu, K., Matsumoto, H.: Spatial positioning measurements up to $150 \mathrm{~m}$ using temporal coherence of optical frequency comb. Precis. Eng. 37, 635-639 (2013).

17. Wei, D., Takamasu, K., Matsumoto, H.: A study of the possibility of using an adjacent pulse repetition interval length as a scale using a helium-neon interferometer. Precis. Eng. 37, 694-698 (2013).

18. Wei, D., Aketagawa, M.: Comparison of length measurements provided by a femtosecond optical frequency comb. Opt. Express. 22, 7040-7045 (2014).

19. W. Sudatham, H. Matsumoto, S. Takahashi and K. Takamasu, "Verification of the positioning accuracy of industrial coordinate measuring machine using optical-comb pulsed interferometer with a rough metal ball target," Precis. Eng. 41, 63-67 (2015)

20. W. Sudatham, H. Matsumoto, S. Takahashi and K. Takamasu, "Non-contact measurement technique for dimensional metrology using optical comb," Measurement 78, 381-387 (2015)

21. Saleh, B.E.A., Teich, M.C.: Fundamentals of Photonics, Wiley Series in Pure and Applied Optics, p. 1177. Wiley-Interscience, Hoboken, N.J (2007), xix)

22. Wei, D., Xiao, M., Yang, P.: Group refractive index calculation by difference approximation for length measurement. J. Eur. Opt. Soc. Rapid Publ. 11, 16013 (2016)

23. Wei, D., Xiao, M., Yang, P.: Selection of numerical differentiation method for calculation of group refractive index of air over all calculable wavelengths. Optik Int. J. Light Electron Opt. 382, 1362-1369 (2017).

24. Butt, R: Introduction to Numerical Analysis Using MATLAB ${ }^{\oplus}$, Infinity Science Series, Jones \& Bartlett Learning, Burlington (2009)

25. Canale, R., Chapra, S: Numerical Methods for Engineers, McGraw-Hill Education, New York City, (2014)

26. Schellekens, P., Wilkening, G., Reinboth, F., Downs, M.J., Birch, K.P., Spronck, J.: Measurements of the refractive index of air using interference refractometers. Metrologia. 22, 279 (1986). 